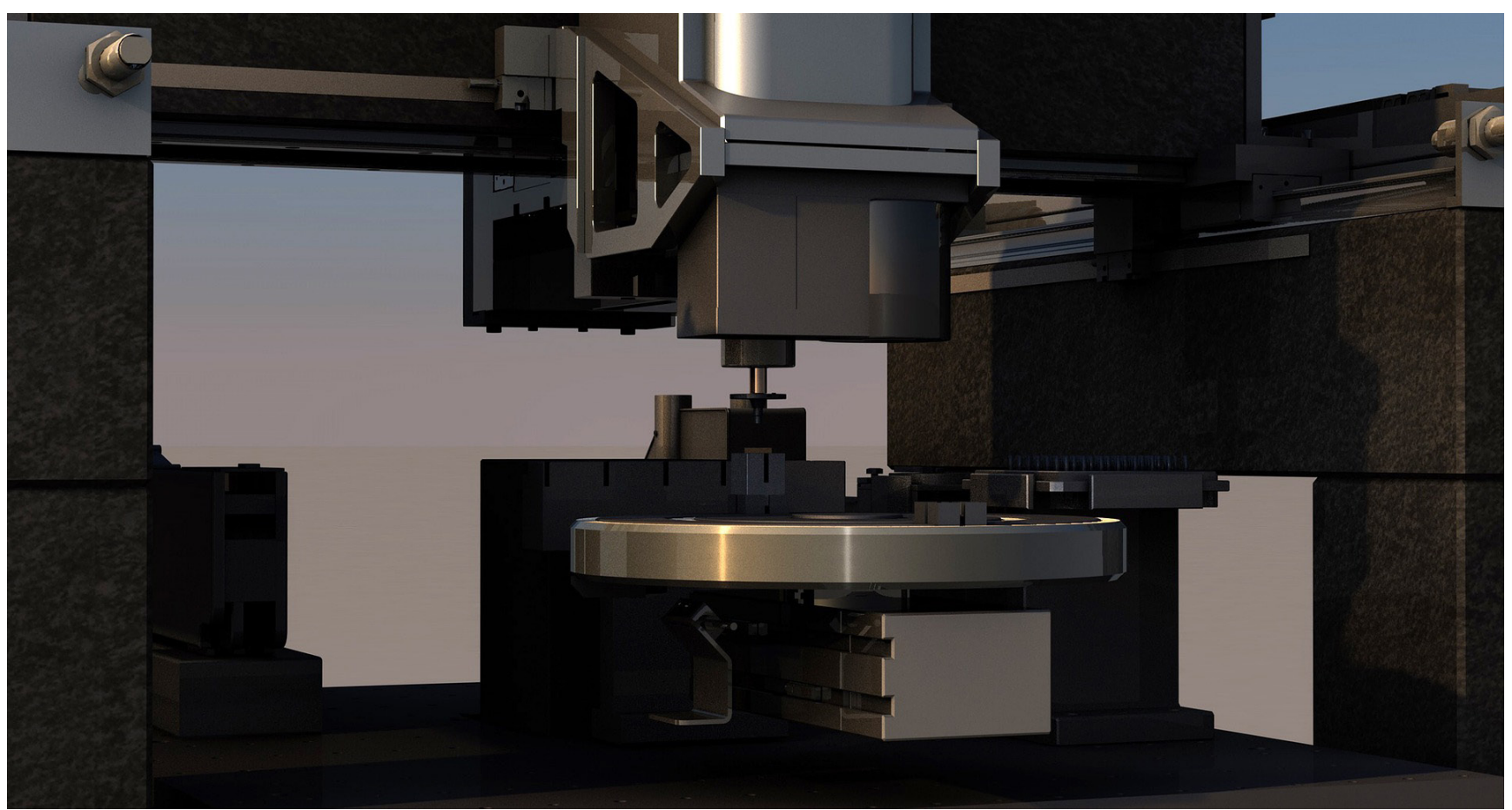

\title{
Comparación entre tecnologías emergentes y tradicionales en automatización e instrumentación industrial
}

\section{Comparison between emerging and traditional technologies in automation and industrial instrumentation}

Alfredy De La Espriella-Babilonia

Especialista, alfredytus@yahoo.com, https://orcid.org/0000-0002-0174-6723,

Universidad Tecnológica de Bolívar, Cartagena de Indias, Colombia. 


\section{Resumen}

El Objeto primordial de este trabajo es demostrar posibilidades con datos reales, y de especificaciones técnicas de los sistemas comerciales de automatización, (tradicionales $y$ emergentes) al microempresario para que pueda actualizar los procesos automatizables, de maquinarias no automatizadas, para ampliar su vida útil y bajar sus costos, e incluir en estas actualizaciones tecnológicas capital humano local, para no tener que adquirir maquinaria nueva usando las tecnologías más adecuadas para cada proceso y para cada situación económica en particular, el carácter emergente de las tecnología conlleva a la búsqueda de la mejor herramienta tecnológica, que permita el usufructo de recurso como lo es el factor tiempo, la economía y la mano de obra profesional, las constante búsqueda de las empresas para ser líderes en su ramo hace que las de carácter obligatorio que las mismas innoven en las herramientas informáticas aprovechando las bondades de las mismas; consiguiente, este artículo esta seccionado de la siguiente manera; en la primera sección se hace una revisión de los mercados y las tecnologías tradicionales y las emergentes para seleccionar unas tecnologías que representen la comparación objeto de este articulo; en la segunda sesión se revisan los datos técnicos más representativos de dichas tecnologías.

Palabras clave: Controlador Lógico Programable (PLC), Controlador de Automatización Programable (PAC)

\section{Abstract}

The main objective of this work is to demonstrate possibilities with real data and technical specifications of commercial automation systems (traditional and emerging) to the microentrepreneur so that he can update the automated processes of non-automated machinery, to extend its life and lower its costs, and to include in these technological updates local human capital, so as not to have to acquire new machinery using the most appropriate technologies for each process and for each particular economic situation, the emerging nature of technology leads to the search for the best technological tool, which allows the use of resources such as time, economy and professional labor, the constant search of companies to be leaders in their field makes it mandatory that they innovate in computer tools taking advantage of the benefits of them; Consequently, this article is divided in the following way; in the first section a review of the markets and traditional and emerging technologies is made to select some technologies that represent the comparison object of this article; in the second session the most representative technical data of these technologies are reviewed.

Keywords: Programmable Logic Controller (PLC), Programmable Automation Controller (PAC) 


\section{2}

\section{Introducción}

En un mundo globalizado, la tecnología de información avanza vertiginosamente en la búsqueda de soluciones rápidas y directas en el ámbito laboral, donde la inclusión de la automatización y la instrumentación industrial en la sociedad es cada vez más evidente y necesaria, es obvio pensar que la misma, está tomando un papel muy importante en la industria moderna, ya que realiza una labor crucial sobre el sistema de calidad, de los productos, bienes y servicios [1].

Autores como [2] expresan es necesario conocer ese mercado que es grande, el impacto que tiene y tendrán los productos emergentes de oriente y otras partes del mundo, con la finalidad de que nuestra industria pueda innovar en sus procesos manuales a procesos automatizados; entre otras cosas.

De igual manera, [3] afirma "que aquellos que no innoven se evaporarían", de ahí un mensaje importante aupando a las grandes empresas a incursionar en las tecnología de información y comunicación; es por ello realizar este documento demostrando todas estas posibilidades con datos reales, y de especificaciones técnicas a los encargados de la actualización tecnológica en cada empresa y al microempresario para que pueda actualizar los procesos automatizables, de maquinarias no automatizadas, pero en buen estado, que tengan buena calidad de producto realizado, para ampliar su vida útil y bajar sus costos, e incluir en estas actualizaciones tecnológicas capital humano local, para no tener que adquirir maquinaria nueva usando las tecnologías más adecuadas para cada proceso y para cada situación económica en particular.

Así mismo, [4] sostienen que "con la evolución tecnológica constante, es natural que las instituciones se enfrenten a cambios drásticos de paradigmas con respecto a las infraestructuras tecnológicas", tomando en cuenta esta premisa, se realiza el presente artículo estructurado de la siguiente manera; en la primera sección se hace una revisión de los mercados y las tecnologías tradicionales y las emergentes para seleccionar unas tecnologías que representen la comparación objeto de este articulo; en la segunda sesión se revisan los datos técnicos más representativos de las tecnologías seleccionadas.

Estado de tecnologías producidas por los fabricantes de productos de automatización.

Según [5] "Afirma que estamos en medio de una revolución es quizás nadar contra la corriente en un océano tormentoso, el malestar en la modernidad quiere efectivamente significar que entramos en un período que muchos denominan post modernidad"; entonces seria propicio indicar que estamos en plena revolución tecnológica, en donde se puede evidenciar que no estar a la par con innovaciones tecnológica puede resultar un olvido en tiempo, dejarían de existir aquellos quienes no incursionan en el mundo global de las TIC.

Por otra parte, [6] señala a la "tecnología como una forma de cultura, que a su vez corresponde a un cierto nivel de desarrollo de lo humano. Siguiendo la historia del desarrollo de las herramientas, llega al momento en que aparece con fuerza la diferenciación entre naturaleza y tecnología, dando lugar a los productos tecnológicos". Por consiguiente, se podría afirmar que las innovaciones tecnológicas pasan a ser parte de vida cotidiana, así como también el poder comprender las modificaciones que estas hacen al mundo. 
Cada vez más, los usuarios finales buscan soluciones, no productos, y esto se aplica para la mayoría de las tecnologías de automatización e innovar, así lo demuestra [2] "el proceso innovador era una actividad que implicaba una estrategia, que requería de un aprendizaje tecnológico por parte de los agentes involucrados y en la que el eje de la actividad de innovación era la empresa", porque las industrias que necesitan automatizar, ahora ponen mayor énfasis en el conocimiento integral de industrias atendidas por sus proveedores, sus capacidades de ingeniería, y su capacidad para proporcionar una solución completa de automatización (PLC, variadores de velocidad, paneles de operador HMI, etc.

Así mismo [7] determina que "las regulaciones modernas buscan liberaral mercado de las telecomunicaciones para que múltiples tecnologías cableadas y no cableadas convivan y se interconecten entre sí", permitiendo así una liberación de la tecnología analógica y dando paso a las nuevas oportunidades que la tecnología de punta ofrece hoy por hoy, esto con respecto a la tecnología de transmisión eléctrica se refiere.

De igual manera, hoy en día el mercado para los PLC está cambiando rápidamente. Los proveedores que quieren tener éxito en este ramo, necesitan entender las necesidades de los clientes y en el mejor de los casos superarlas [7] afirma que "las comunicaciones por las redes eléctricas, con cobertura mayor que las de telecomunicaciones en zonas tradicionalmente no económicas, podrían reducir la brecha digital en países en desarrollo".

Ejemplos de tecnologías.

A continuación en las figuras de la 1 a la 6 se pueden observar algunos ejemplos de tecnologías:

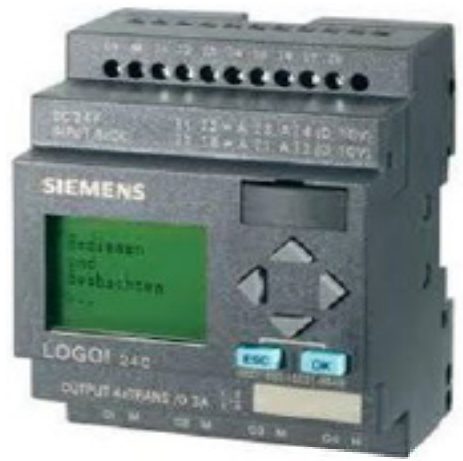

Figura 1. LOGO! de Siemens

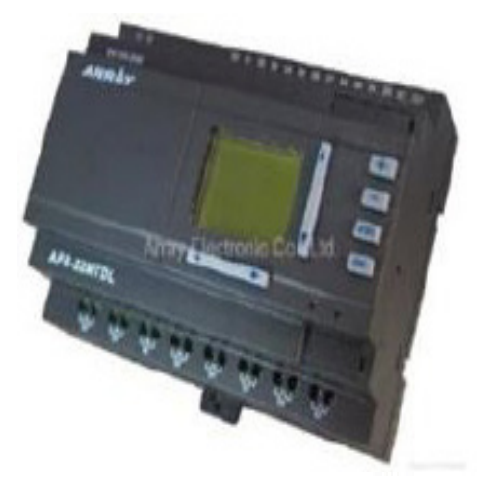

Figura 2. APB de Array electronics

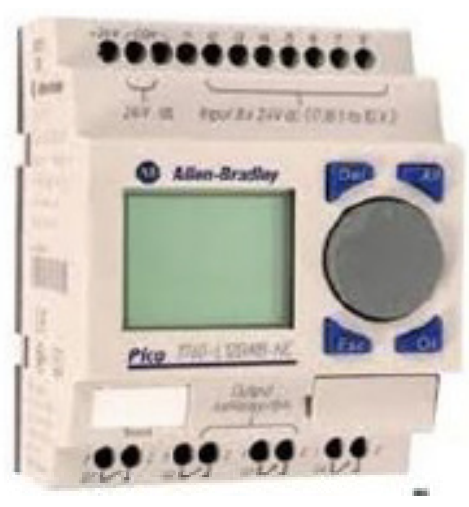

Figura 3. EASY de Moeller 


\section{4}

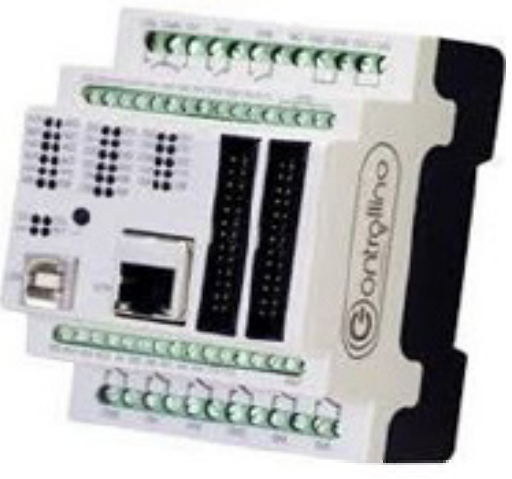

Figura 4. CONTROLLINO de Gsd

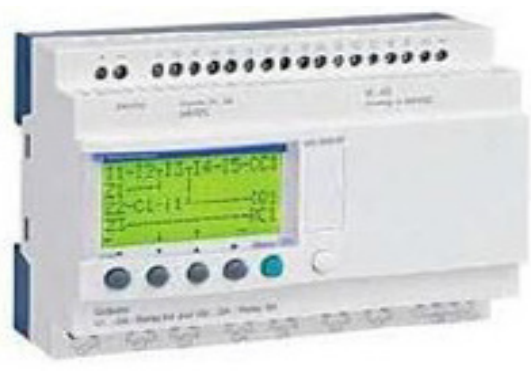

Figura 5. ZELIO de Schneider Electric

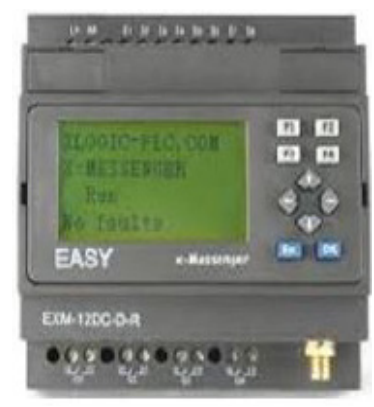

Figura 6. EASY de Xlogic

Comparación técnica de las tecnologías seleccionadas

Actualmente somos testigos de un cambio de paradigma, así lo releja "La profunda transformación digital, a raíz de la globalización y su cambio paradigmático está impactando especialmente en la economía y sus empresas actuales" [8]; "apuntado a una digitalización paulatina de la cultura esto no ha hecho sino incrementarse drásticamente en la última década" [9]; por lo tanto, escoger una tecnología se hace cada vez más difícil, ya que las bondades son inmensurables, sin embargo, se escoge según las necesidades varios tipos de tecnología para su debida comparación.

De igual manera [9] refleja que "Las tecnologías de la información son el último eslabón de la cadena de inventos humanos que va desde el lenguaje hablado, la escritura y la imprenta, hasta nuestros días", generalizando así la incorporación de las TIC en el ámbito laboral, propiciando una competitividad inminente entre los creadores se software a nivel empresarial, estos cambios tecnológicos han ampliado las perspectiva de trabajo en las empresas, creando modelos innovadores para los procesos de telecomunicaciones, redes digitales y otros.

Por su parte, [7] afirma que "las telecomunicaciones se han abierto a la existencia de múltiples tecnologías. Unas son las comunicaciones por cable eléctrico, - PLC/BPL. Las comunicaciones por las redes eléctricas, con cobertura mayor que las de telecomunicaciones podrían reducir la brecha digital en países en desarrollo", sin embargo, no están exentas de regularizaciones eléctricas y digitales de acuerdo al país en que sean usadas, así lo sugiere [10] "la promoción de la entrada de nuevas tecnologías deben regularse", sin embargo, más allá de restringir lo que se quiere con estas restricciones es el acceso universal de las tecnologías cableadas y no cableadas; para nada puede ser un secreto que esta tecnología abre brechas gigantes para la transmisión de datos digitales en altas velocidades.

Comparación de lenguajes de programación

Por consiguiente, para hacer una comparación técnica ecuánime entre las marcas elegidas se comparan varias características de los mismos, como lo es la comparativa de lenguajes; con 
esta característica vemos que tan normalizado está el PLC (Procesador Lógico Programable) en cuanto a programación, en comparación con IEC (Comisión Electrotécnica Internacional), Esta tabla denota las múltiples vías en las cuales puede resolverse la situación problemática en cada PLC en Particular. "Quienes apuesten por estas herramientas estarán en una mejor posición para lograr un lugar privilegiado en el futuro que hoy ya se empieza a diseñar" [1112].

Tabla 1: Comparativa de Lenguajes Usados Vs Estándar IEC 1131

\begin{tabular}{|c|c|c|c|c|c|c|}
\hline & $\begin{array}{c}\text { Siemen } \\
\mathrm{s}\end{array}$ & $\begin{array}{c}\text { ARRA } \\
\mathbf{Y} \\
\end{array}$ & $\begin{array}{c}\text { Moelle } \\
\mathrm{r}\end{array}$ & $\begin{array}{c}\text { Gsd } \\
\text { Tronic }\end{array}$ & $\begin{array}{c}\text { Schneid } \\
\text { er }\end{array}$ & $\begin{array}{c}\text { XLOGI } \\
\mathrm{C}\end{array}$ \\
\hline $\begin{array}{l}\text { LENGUAJE DE } \\
\text { PROGRAMACION }\end{array}$ & LOGO! & APB & EASY & $\begin{array}{c}\text { Controli } \\
\text { no }\end{array}$ & ZELIO & EASY \\
\hline $\begin{array}{l}\text { Diagrama De } \\
\text { Contactos (KOP, } \\
\text { LD) }\end{array}$ & 1 & 1 & 1 & & 1 & \\
\hline $\begin{array}{l}\text { Esquema De } \\
\text { Bloques De } \\
\text { Funciones (FBD) }\end{array}$ & 1 & 1 & 1 & & 1 & 1 \\
\hline $\begin{array}{l}\text { Diagrama } \\
\text { funcional de } \\
\text { secuencias } \\
\text { (GRAFCET) }\end{array}$ & & & & & 1 & \\
\hline $\begin{array}{l}\text { Lista de } \\
\text { instrucciones (IL) }\end{array}$ & & & & & & \\
\hline $\begin{array}{l}\text { Texto } \\
\text { Estructurado } \\
\end{array}$ & & & & 1 & & \\
\hline SUMATORIAS & 2 & 2 & 2 & 1 & 3 & 1 \\
\hline
\end{tabular}

Comparativa tecnológica de software

por medio de esta podemos darnos cuenta que tan útil es el software en la programación y operación del controlador, ya que con esto se resuelven fallas y se encuentran los elementos defectuosos en la operación del mismo. Además es importante que el mismo disponga de un simulador del software realizado con el objetivo de probar la programación en la misma forma en que fue hecho el programa (tabla 2)

Tabla 2: Comparativa Tecnológica de Software.

\begin{tabular}{|l|c|c|c|c|c|c|}
\hline & LOG0! & APB & EASY & no & ZELI0 & EASY \\
\hline SIMULADOR & 1 & 1 & 1 & & 1 & 1 \\
\hline Prueba En Linea & 1 & 1 & 1 & & 1 & 1 \\
\hline SUMAIORIAS & 2 & 2 & 2 & 0 & 2 & 2 \\
\hline
\end{tabular}




\section{6}

Comparativa de precios

Los precios de estos controladores en Colombia son variantes pero estos los hemos conseguido con cada uno de los proveedores para el año 2015 (tabla 3).

Tabla 3. Comparativa De Precios En Pesos Colombianos.

\begin{tabular}{|c|c|c|c|c|c|}
\hline L0G0! & APB & EASY 700 & Controlin0 & ZELI0 & EASY \\
\hline & & & $6 \mathrm{Ry} 0$ ut, & & \\
\hline & $8 \mathrm{In} / 4$ & & $8 \mathrm{x} \mathrm{An/Di} \mathrm{In}$ & & $8 \mathrm{In} / 4$ \\
\hline $8 \mathrm{In} / 4$ 0ut & 0 ut & 12 in $/ 6$ out & $8 \mathrm{Di} 0$ ut & $8 \mathrm{In} / 40$ ut & 0 ut \\
\hline $\mathbf{7 9 8 . 8 0 0}$ & $\mathbf{4 3 0 0 0 0}$ & 1.462 .600 & 600000 & 935000 & 400000 \\
\hline
\end{tabular}

\section{Conclusiones}

Definitivamente las tecnologías emergente es una realidad palpable e inminente en este mundo globalizado, como quedó demostrado a los largo de este artículo, dejando claras las opciones de automatización que hay en el mercado, comparándolas técnica y económicamente para que el lector tenga una visión más detallada del entorno, y así pueda acceder de una forma más fluida a este tipo de tecnología en sus procesos análogos.

En cuanto a la parte técnica realmente existen ligeras diferencias que harían de la selección no un tema técnico sino uno económico, de mantenimiento, y de logística cuando necesiten un reemplazo o un módulo adicional, así como también la estrecha relación entre las tecnologías emergentes y la innovación tecnológica influyendo en los procesos empresariales, dándole calidad al producto o servicio ofrecido al cliente.

Finalmente, en el marco de las nuevas tecnologías emergente y el desarrollo industrial, se es necesario siempre fomentar la importancia de los controladores lógicos programables ya que los mismos permitirán productiva con eficiencia y eficacia, fiabilidad en los resultados y un mayor control de los resultados, es importante tener en cuenta que esta tecnología no solo está presente en las industrias sino en nuestra cotidianidad como lo es nuestros hogares.

\section{Referencias}

[1] J. Fajardo, "Virtualización de la producción académica en la Facultad de Arquitectura de la Universidad de Cuenca (Ecuador). ASRI - Arte y Sociedad", Revista de Investigación. no. 7, 2014.

[2] I. Vega, "Innovación, tecnologías emergentes y cambio organizacional. El caso de la Fórmula 1", Revista Venezolana de Análisis de Coyuntura, vol. XXIV, núm. 1, 2018 
[3] J. Higgins, "Innovate or evaporate: Seven secrets of innovative corporations", Revista The Futurist, vol 29, no. 5, 1995

[4] J. Rojas, J. Forero, C. Montenegro y P. Gaona, "Hacia la virtualización de escritorios para la entrega de ambientes académicos basados en DaaS", Revista Logos, Ciencia \& Tecnología, vol. 7, núm. 2, pp. 85-96, enero-junio, 2016

[5] F. Mires, La revolución que nadie soñó o la otra postmodernidad. Caracas: Editorial Nueva Sociedad. 1996

[6] M. Mejía, "La tecnología, la(s) cultura(s) tecnológica(s) y la educación popular en tiempos de globalización", Polis Revista Latinoamericana, 2004. [En línea], Disponible en: http://journals.openedition.org/polis/6242

[7] A. Ibarra y E. Castruita, "Tecnologías PLC/BPL en México y sus retos para las regulaciones: ¿existen oportunidades de desarrollo?", Revista gestión y política pública, vol. xiv, no. 2, 2005

[8] A. Sánchez, "Galeato pro universitas humanitatis", Revista Electrónica de Pensamiento, Economía y Sociedad, vol. 20, pp. 19-36, 2003

[9] F. Sáez, "TVIC: Tecnologías para la vida cotidiana”, TELOS, vol. 73, pp. 4-6, 2007

[10] Y. Rabeau, Y, "The Shumpeterian Wave in Telecommunications: Public Policy Implications", Choices, vol. 10, núm. 7, pp. $5-11,2007$

[11] J.M. Spector, "Emerging Educational Technologies and Research Directions", Journal of Educational Technology \& Society, vol. 16, no. 2, pp. 21-30, 2007

[12] H. Páez, R. Zamora y J. Bohórquez, "Programación de Controladores Lógicos (PLC) mediante Ladder y Lenguaje de Control Estructurado (SCL) en MATLAB', Fac. Ing, vol. 24, no. 39, pp. 109- 119, Mayo-Ago. 2015. 